\title{
Pseudo-anaphylaxis to Polyethylene Glycol (PEG)-Coated Liposomes: Roles of Anti-PEG IgM and Complement Activation in a Porcine Model of Human Infusion Reactions
}

Gergely Tibor Kozma, ${ }^{\dagger}{ }^{\ddagger}$ Tamás Mészáros, $^{\dagger}$ Ildikó Vashegyi, $^{\ddagger}$ Tamás Fülöp, ${ }^{\dagger}$ Erik Örfi, ${ }^{\dagger}$ László Dézsi, $^{\dagger}$ László Rosivall, ${ }^{\dagger, \ddagger}{ }^{\ddagger}$ Yaelle Bavli, ${ }^{\|}$Rudolf Urbanics, ${ }^{\dagger, \ddagger}$ Tom Eirik Mollnes, ${ }^{\perp, \#, \text { IL }}$ Yechezkel Barenholz, ${ }^{\|, \square}$

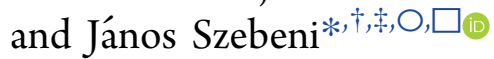

${ }^{\dagger}$ Nanomedicine Research and Education Center, Semmelweis University, Budapest 1089, Hungary

${ }^{\ddagger}$ SeroScience Ltd., Budapest 1125, Hungary, and Cambridge, Massachusetts 02138, United States

${ }^{\S}$ Department of Pathophysiology, International Nephrology Research and Training Center, Semmelweis University, Budapest 1089, Hungary

"Laboratory of Membrane and Liposome Research, IMRIC, Hebrew University-Hadassah Medical School, Jerusalem 9112102, Israel

${ }^{\perp}$ Department of Immunology, Oslo University Hospital, Rikshospitalet, Oslo 0372, Norway

"Research Laboratory, Nordland Hospital Bodø, and Faculty of Health Sciences and TREC, University of Tromsø, Tromsø 9019, Norway

${ }^{\text {II }}$ Centre of Molecular Inflammation Research, Norwegian University of Science and Technology, Trondheim 7012, Norway

${ }^{\circ}$ Department of Nanobiotechnology and Regenerative Medicine, Faculty of Health, Miskolc University, Miskolc 3515, Hungary

S Supporting Information

ABSTRACT: Polyethylene glycol (PEG)-coated nanopharmaceuticals can cause mild to severe hypersensitivity reactions (HSRs), which can occasionally be life threatening or even lethal. The phenomenon represents an unsolved immune barrier to the use of these drugs, yet its mechanism is poorly understood. This study showed that a single i.v. injection in pigs of a low dose of PEGylated liposomes (Doxebo) induced a massive rise of anti-PEG IgM in blood, peaking at days 7-9 and declining over 6 weeks. Bolus injections of PEG-liposomes during seroconversion resulted in anaphylactoid shock (pseudo-anaphylaxis) within 2-3 min, although similar treatments of naïve animals led to only mild hemodynamic disturbance. Parallel measurement of pulmonary arterial pressure (PAP) and sC5b-9 in blood, taken as

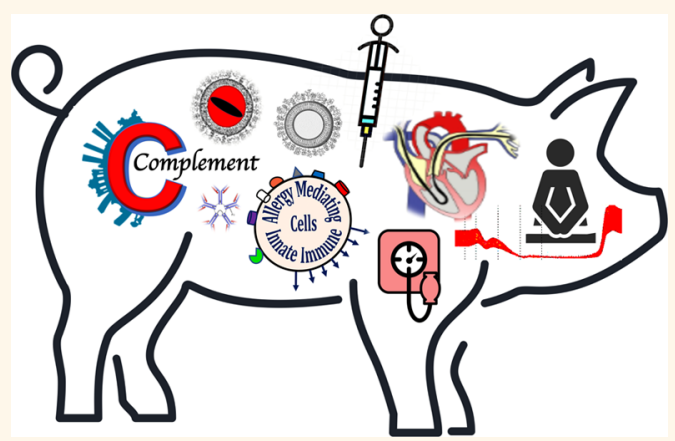
measures of HSR and complement activation, respectively, showed a concordant rise of the two variables within $3 \mathrm{~min}$ and a decline within $15 \mathrm{~min}$, suggesting a causal relationship between complement activation and pulmonary hypertension. We also observed a rapid decline of anti-PEG IgM in the blood within minutes, increased binding of PEGylated liposomes to $\mathrm{IgM}^{+} \mathrm{B}$ cells in the spleen of immunized animals compared to control, and increased C3 conversion by PEGylated liposomes in the serum of immunized pigs. These observations taken together suggest rapid binding of anti-PEG IgM to PEGylated liposomes, leading to complement activation via the classical pathway, entailing anaphylactoid shock and accelerated blood clearance of liposome-IgM complexes. These data suggest that complement activation plays a causal role in severe HSRs to PEGylated nanomedicines and that pigs can be used as a hazard identification model to assess the risk of HSRs in preclinical safety studies.

KEYWORDS: allergy, liposomes, anaphylatoxins, animal models, immunoglobulins, anaphylactoid shock, pulmonary hypertension

$\mathrm{P}$ olyethylene glycols (PEGs), highly hydrated bulky polymers in the $0.5-40 \mathrm{kDa}$ range, are widely used to improve the therapeutic efficacy of drug carrier liposomes and proteins by extending their circulation time.
Received: May 21, 2019

Accepted: July 26, 2019

Published: July 26, 2019 
An unresolved issue with such PEGylated nanopharmaceuticals is their recognition by the immune system manifested in non-IgE-mediated (pseudoallergic) hypersensitivity reactions (HSRs), an adverse event commonly called infusion reaction. ${ }^{1-7}$ PEGylated nanomedicines that have been reported to cause such reactions include PEGylated liposomal doxorubicin (Doxil/Caelyx), ${ }^{8}$ PEGylated G-CSF (pegfilgrastim, Neulasta), ${ }^{9}$ PEGylated erythropoetin (mono-mPEG-epoetin- $\beta$, Mircera), ${ }^{10}$ PEGylated recombinant human factor VIII (Adynovate), ${ }^{11}$ and PEGylated phenylalanine ammonia lyase (pegvaliase-pqpz, Palynziq), ${ }^{12}$ with at least three formulations withdrawn from clinical use partly because of severe HSRs: PEGylated EPOmimetic peptide (peginesatide, Omontys), ${ }^{13}$ PEGylated urate oxidase (pegloticase, Krystexxa), ${ }^{14}$ and a PEGylated IXa blocker RNA aptamer (pegnivacogin, Revolixys). ${ }^{15}$

The symptoms include shortness of breath, facial redness and swelling, chest pain, back pain, flashing, rash, chills, panic, and fever that mostly arise shortly after the first treatment, although reactions starting later or after repeated treatments are also observed. In most cases the problem spontaneously resolves or can easily be controlled; however, occasionally, the reaction can escalate into pseudo-anaphylaxis, a severe, occasionally lethal form of infusion reactions resembling anaphylaxis without $\operatorname{IgE}$ playing a role. It is also known as anaphylactoid reaction, or anaphylactoid shock.

Such HSRs with occasional pseudo-anaphylaxis are not limited to PEGylated drugs but arise from a great variety of i.v. medications, including micellar drugs, contrast agents, biologicals, enzyme therapies, iron compounds, and even small molecules. ${ }^{3,7}$ The phenomenon presents substantial clinical, regulatory, and industrial concern, yet its immune mechanism is far from being understood more than 100 years after the discovery of anaphylaxis. ${ }^{5}$ Prediction of severe reactions is uncertain at present, and their prevention and treatment are still empirical and symptomatic. $^{1-7}$

In order to better understand these reactions and help their prediction and prevention, the present study used the porcine complement activation-related pseudoallergy (CARPA) model, $^{16-19}$ which was shown previously to display typical symptoms of cardiac anaphylaxis, ${ }^{16}$ whereupon the cause of sudden death is shock with cardiac arrest. We used the model for uncovering the immune mechanism underlying the HSRs to PEGylated drugs, taking PEGylated liposomal doxorubicin (Doxil/Caelyx) and its drug-free formulation, placebo Doxil (Doxebo) $^{20}$ as model agents. By providing evidence for a causal relationship among the induction of anti-PEG antibodies, complement activation, and pseudo-anaphylaxis, the present data highlight the mechanism of Doxil-induced severe HSRs and use of the pig model in understanding and predicting this type of immune toxicity of PEGylated nanopharmaceuticals.

\section{RESULTS AND DISCUSSION}

Immunogenicity of Doxebo. A single i.v. treatment of pigs with low (0.1 mg phospholipid (PL)/kg) Doxebo led to a massive rise of anti-PEG IgM and a parallel smaller rise of antiPEG IgG antibodies (Abs) (Figure 1). Panels A and B show the titers on linear and logarithmic scales, respectively, the latter amplifying the lower region of titer changes. The rise of Abs started on day 3 following treatment, and the titers reached a peak between days 7-9 and returned to close to baseline after 4-6 weeks. Essentially similar, although less expressed titers were obtained when whole Doxebo was used as antigen in the ELISA (data not shown).

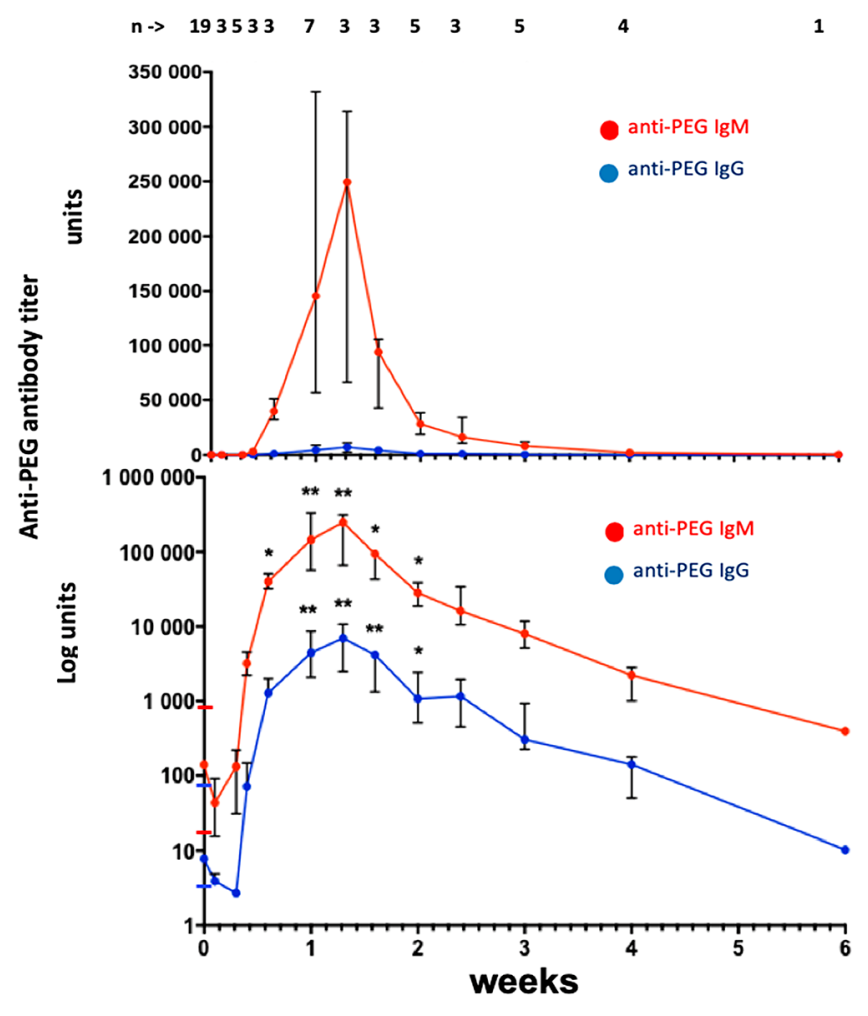

Figure 1. Immunogenicity of Doxebo with anti-PEG $\mathrm{Ab}$ titers presented on both linear (A) and logarithmic (B) scales. Units are defined in the Materials and Methods. Points represent median \pm interquartile range (IQR); titers from $n$ pigs specified above the curves at each time point in panel $A$. The $n=19$ value at baseline is explained by our combination of $\mathrm{Ab}$ measurements in immunized ( $n$ $=12)$ and nonimmunized $(n=7)$ pigs only at this time. Nonimmunized pigs showed no elevation of Ab titers. Subjecting these data to the Kolmogorov-Smirnov test did not show a Gaussian distribution of titers at all days, hence the median $\pm I Q R$ presentation. The Kruskal-Wallis test followed by Dunn's multiple comparison indicated a significant difference relative to baseline on each day from 3 to $14(*, P<0.05$ or $* *, P<0.01)$. Details of the ELISA are described in the Materials and Methods.

A further observation in these experiments (visible only in Figure 1B) was that the anti-PEG IgM/IgG levels were not zero at baseline (time 0 ), indicating the presence of pre-existing (natural) anti-PEG antibodies. The anti-PEG IgM titer substantially varied in animals within the 20-1000 unit range, IgM being in an order of magnitude higher range than IgG (Figure 1B, $y$ axis, color-matched error bar endings). Yet another unexpected finding in Figure 1B was that the initial titers of both anti-PEG IgM and IgG dropped at days 1-2 after immunization with Doxebo.

Clearance of anti-PEG IgM Has the Same Time Course as That of Infusion Reactions. The experiment in Figure $1 \mathrm{~B}$ showed the decline of anti-PEG IgM and IgG within a day following immunization with Doxebo, although the exact kinetics of this decline remained unresolved on a shorter time course. Since the HSR to Doxil occurs on a time scale of minutes, ${ }^{20}$ a question of particular interest to explore was the possible coincidence of these changes. Accordingly, in the next series of experiments naïve pigs were bolus-injected with Doxil, and we measured both the rise of pulmonary arterial pressure (PAP) (a measure of HSR, Figure 2A) and the level anti-PEG IgM in the blood (Figure $2 \mathrm{~B}$ and $\mathrm{C}$ ) on the minute time scale. In keeping with earlier data ${ }^{20}$ bolus injection of Doxil led to a 

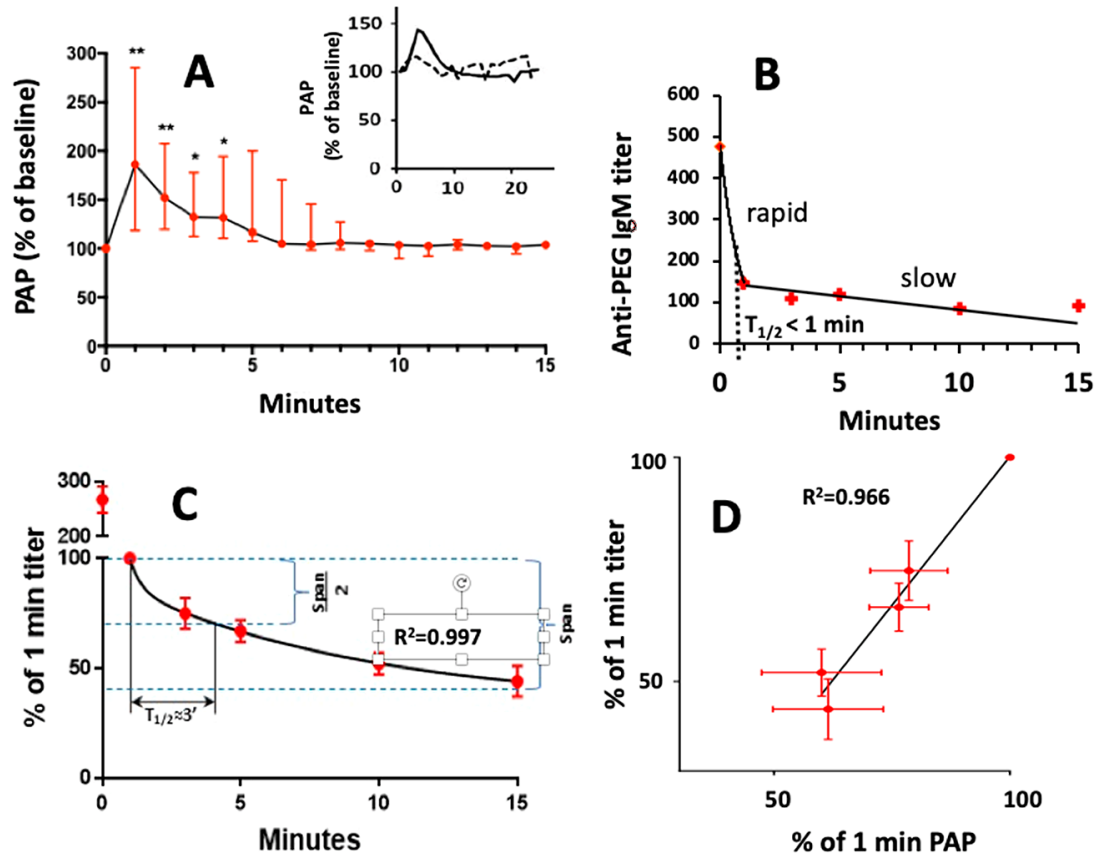

Figure 2. Hemodynamic changes and plasma anti-PEG IgM levels in (naïve) pigs injected with PEGylated liposomes. (A) Pulmonary hypertensions caused by bolus injection of $0.1 \mathrm{mg}$ PL/ $\mathrm{kg}$ Doxil. Points represent median $\pm \mathrm{IQR}, \boldsymbol{n}=5$. Friedman test followed by Dunn's multiple comparison test showed the 1-4 min values were significantly different from baseline $(* *, P<0.01$ or $*, P<0.05)$. The inset exemplifies the effect of Doxebo, reported in ref 20. (B) Plasma anti-PEG IgM titers as a function of time following injection of $0.1 \mathrm{mg} / \mathrm{kg} \mathrm{Doxil.}$ Typical values in 1 of 5 pigs. (C) Plasma anti-PEG IgM following injection of $0.1 \mathrm{mg} / \mathrm{kg}$ Doxil, \% of $1 \mathrm{~min}$ readings, mean \pm SEM, $n=5$. One phase logarithmic curve fitting gave an $R^{2}$ of 0.997 , which is highly significant. (D) Correlation between the relative changes of anti-PEG IgM titer and pulmonary arterial pressure following injection of $0.1 \mathrm{mg} / \mathrm{kg}$ Doxil, with the $1 \mathrm{~min}$ readings taken as baseline. Values are mean \pm SEM, $n=5$. Pearson $R^{2}=0.966$ indicates highly significant correlation.

significant rise of PAP within $2 \mathrm{~min}$, and the values returned to baseline within 7-10 min (Figure 2A). Similar treatment of animals with Doxebo in an earlier study ${ }^{20}$ had similar results, although milder pulmonary reaction (inset in Figure 2A). Of great surprise, the blood levels of anti-PEG IgM fell in the same time window as the HSR occurred with clear separation into two phases; a rapid plunge within 1 min was followed by a slow decline over $15 \mathrm{~min}$ (Figure 2B). The latter process was consistent and reproducible in 5 pigs (Figure 2C), allowing highly significant logarithmic curve fitting $\left(R^{2}=0.997\right)$ typical of first-order biological reactions. Importantly, correlating the antiPEG IgM titer changes with pulmonary hypertension also showed a highly significant correlation $\left(R^{2}=0.97\right.$, Figure $\left.2 \mathrm{D}\right)$. These data taken together provide quantitative evidence for a causal relationship between the decline of naturally occurring anti-PEG IgM and Doxil-induced HSRs.

Amplified Reactogenicity of PEGylated Liposomes in Pigs Immunized with Doxebo. If anti-PEG IgM plays a causal role in Doxil-induced HSRs, as suggested by the data in Figure 2, intentional elevation of these Abs by way of immunization with Doxebo (Figure 1) is expected to increase the reactogenicity of PEGylated liposomes. The experiments shown in Figure 3 proved this prediction with unexpected power. Bolus injections of Doxebo and Doxil in 5 immunized pigs led within 2 min to life-threatening HSR in one and lethal pseudo-anaphylaxis requiring resuscitation in 4 animals, regardless of the timing of the test within the 2-6-week window of seroconversion. As illustrated with the surviving animal in Figure 3A, the PAP considerably increased in both amplitude and duration compared to that seen in naïve animals (Figure $2 \mathrm{~A})$, and we observed significant SAP and heart rate (HR) changes even after repeated injections of liposomes (Figure 3A). This implies loss of tachyphylaxis (self-tolerance), which is a characteristic feature of Doxil reactions in naïve pigs and is thought to be due to depletion of natural antibodies, ${ }^{20}$ for which we obtained evidence in Figure $2 \mathrm{~B}$ and $\mathrm{C}$. Thus, the consumption of reactive antibodies during the first reaction was not enough to deplete them to nonreactive levels. Figure $3 \mathrm{~B}$ illustrates the deadly pseudoanaphylaxis with maximal rise of PAP and shock developing within $2 \mathrm{~min}$. The panel also illustrates the moments of resuscitation with cardiac massage and epinephrine, resulting in an overshot of SAP. Figure 3C shows that these severe reactions were associated with extensive flushing and rash within $3 \mathrm{~min}$.

Regarding the dose-effect relationship between anti-PEG IgM and HSRs in immunized pigs, apart from the need or no need for resuscitation, the reactions were quantitatively indistinguishable regardless of the actual-significantly elevated-titers of anti-PEG IgM at the time of the experiment. This suggests that the significant correlation between anti-PEG IgM and HSR obtained for naïve animals (Figure 2D) is not applicable in immunized pigs, as the amount of anti-PEG IgM in blood far exceeds the plateau of the dynamic range of doseeffect relationship, whose exact limits remain to be determined.

Evidence for a Role of Complement Activation in PEGylated Liposome-Induced Pseudo-Anaphylactic Shock in Immunized Pigs. The data in Figure 3 reveal effective sensitization of animals for PEGylated liposomeinduced HSRs by immunization-induced rise of anti-PEG $\operatorname{IgM}$, which is consistent with a causal role of these Abs in the phenomenon. As for the exact mechanism of the cause-effect relationship, considering that antigen-bound $\operatorname{IgM}$ is one of the 


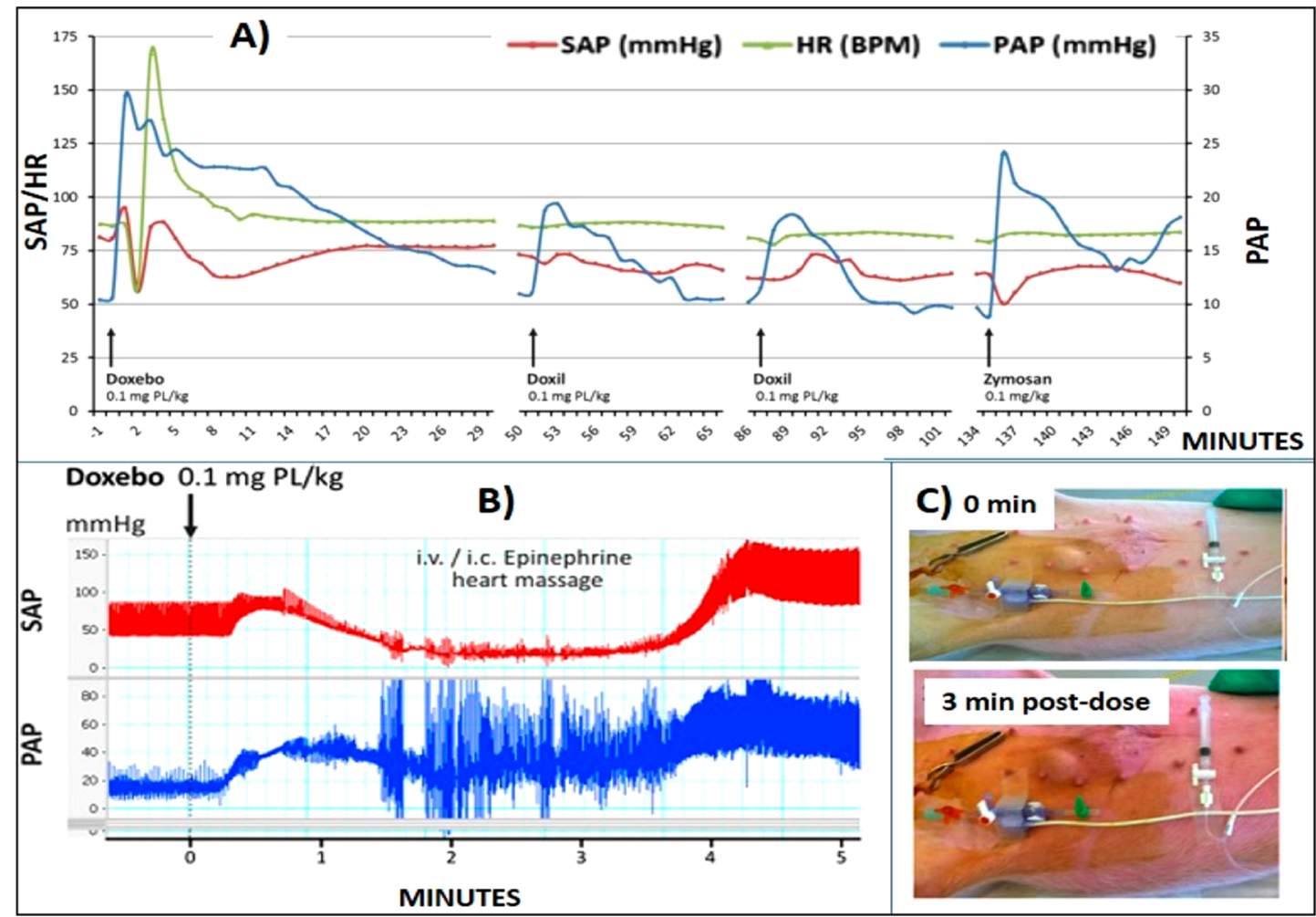

Figure 3. Reactogenicity of PEGylated liposomes in pigs immunized with Doxebo. (A) Hemodynamic changes in one out of 5 animals, showing severe but not lethal responses to repetitive injections of the specified liposome doses 4 weeks after immunization. (B) pseudo-anaphylactic shock in another pig illustrating the reaction of 4 out of 5 immunized pigs tested in the 2-6-week interval after immunization. In real-time hemodynamic tracings, the large-amplitude noise coincided with cardiac massage. (C) Abdominal region of the skin before ("control") and 3 min after the injection of Doxebo, showing confluent skin flushing and rash observed in all 5 pigs. The objects in the pictures include a three-way stopcock connected to a pressure transducer and the syringe used to inflate the spinnaker balloon of the Swan-Ganz catheter (see Materials and Methods).
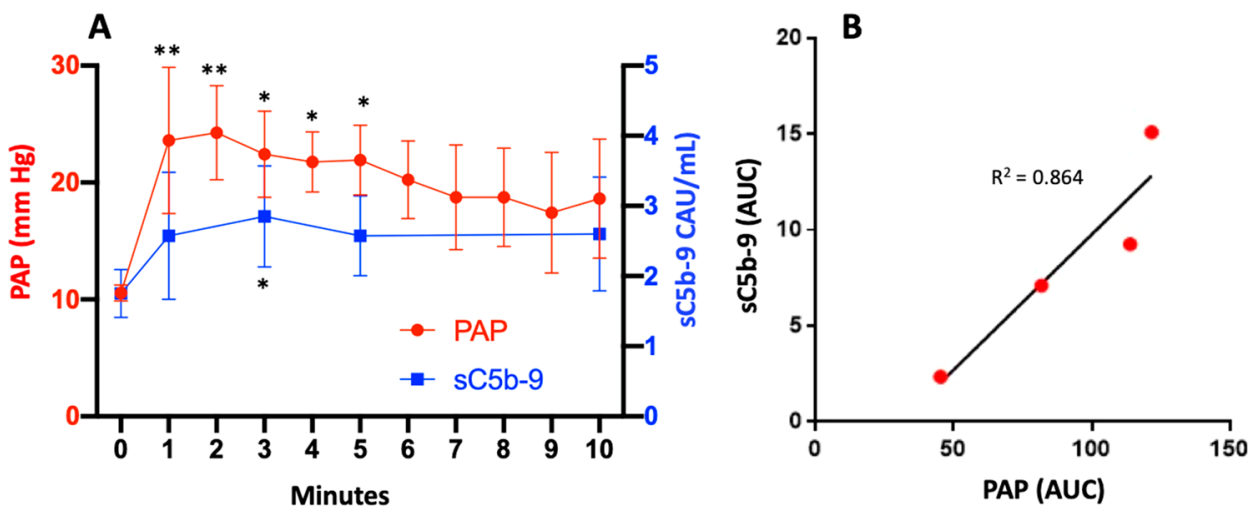

Figure 4. Doxebo-induced complement activation (sC5b-9) and pulmonary hypertension (PAP) in pigs immunized with Doxebo. (A) Time course of PAP (red) and concurrent sC5b-9 (blue) changes following liposome challenge. Points represent median \pm IQR in 4 pigs where blood samples could be collected. Both PAP and sC5b-9 values were statistically different from their baseline $(0 \mathrm{~min})$ at $* P<0.05$ and $* * P<0.01$, obtained with the Friedman test followed by Dunn's multiple comparison test. (B) Correlation between the area under the curve (AUC) values of PAP and sC5b-9 during $10 \mathrm{~min}$ by the Pearson correlation test $\left(P=0.07, R^{2}=0.864\right)$.

most powerful activators of the complement system via the classical pathway, and that there is a large body of evidence for the CARPA theory, i.e., that complement activation plays a causal role in many HSRs (Supporting Table 1), complement activation represents an obvious link between anti-PEG IgM and HSR. However, such a link has had only indirect experimental support to date, and the elevation of complement activation byproducts in pig blood during HSRs has never been shown. $^{16-21}$
To fill this gap of information, we measured the levels of porcine soluble complement terminal complex (sC5b-9) in the plasma of immunized pigs during the first Doxebo-induced massive hemodynamic disturbance (Figure 3A and B). Figure $4 \mathrm{~A}$ shows that the pulmonary hypertension in these animals was closely paralleled by a rise of sC5b-9 in blood, and, consistent with the fact that $\mathrm{sC} 5 \mathrm{~b}-9$ is the final product of the complement activation cascade, its peak at about 3 min was slightly delayed relative to the peak of the PAP curve. At this time the rise of 
sC5b-9 was significant relative to baseline, and the correlation between PAP and sC5b-9 was strong when the areas under the curve (AUC) of complement and PAP responses were analyzed by linear regression (Figure 4B). These data therefore support a causal relationship between complement activation and HSR.

Ex Vivo Evidence for Splenic Production of LiposomeBinding Anti-PEG IgM. Further questions raised by the above results are the organ source of anti-PEG IgM and the mechanism of its rapid clearance. In an experiment addressing these questions we prepared spleen cell suspensions from an immunized and a control pig on day 9 postimmunization and measured by flow cytometry the binding of Doxil to $\operatorname{IgM}^{+} \mathrm{B}$ cells in vitro. The clearly discernible increase of Doxil ${ }^{+}$cells in the upper right gates in Figure 5C vs A indicates the binding of Doxil

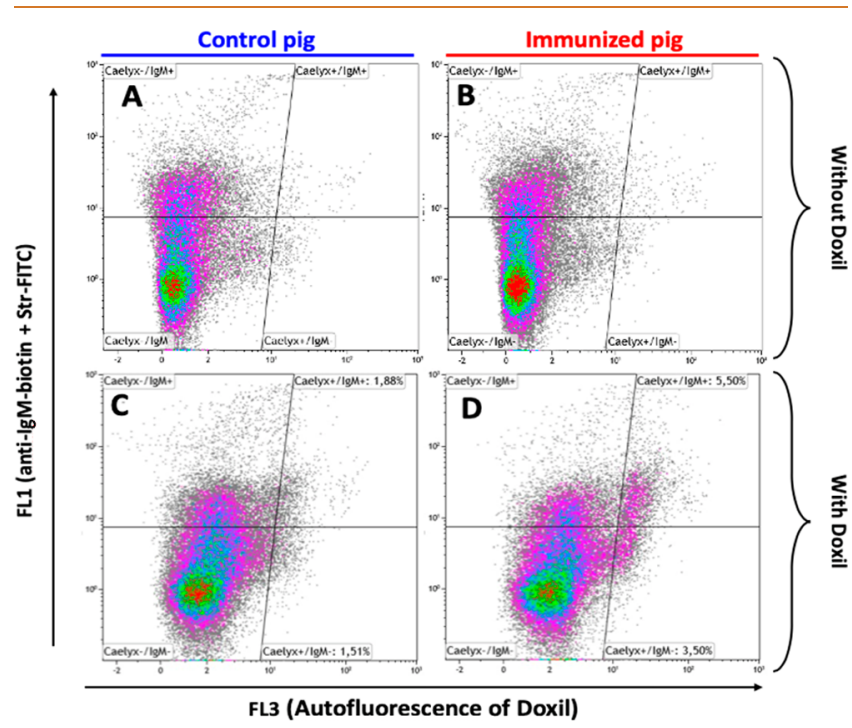

Figure 5. Effect of immunization with Doxebo on Doxil binding to splenic B cells. Spleen cells taken from an immunized and a control animal 9 days after immunization were exposed to FITC-labeled anti-IgM and fluorescent Doxil in vitro, and the fraction of cells binding FITC-IgM and/or Doxil were determined in a flow cytometer. Panels A-D show the distribution of cells displaying different degrees of IgM and Doxil-specific fluorescence in immunized and control animals. The upper right quadrant is a gate on $\mathrm{IgM}^{+} /$Doxil $^{+}$cells. Str-FITC, streptavidin-FITS

to $\operatorname{IgM}^{+}$cells even in control animals. Most noticeably, the cell count of $\mathrm{IgM}^{+} /$Doxil $^{+}$cells tripled in immunized animals vs control (Figure 5D vs C), indicating significant proliferation of Doxil-binding cells in immunized pigs. These observations are consistent with the presence of Doxil-binding anti-PEG IgM on the surface of normal (naïve) porcine splenocytes and significant increase of these cells following immunization. Liberation of these Abs into the circulation in naïve animals may be one source of natural anti-PEG IgM in the blood of pigs, while the increased release of these Abs into the blood explains the elevated anti-PEG IgM in immunized pigs. Furthermore, the binding of Doxil to these Abs on B cells provides evidence for the capability of Doxebo/Doxil to bind to these Abs in the circulation. These findings and explanations taken together support the proposal that the binding of anti-PEG IgM to Doxebo/Doxil in blood explains complement activation, which entails anaphylatoxin production and opsonization of liposomes. The former process explains the HSRs, while the latter is consistent with the first-order removal of anti-PEG IgM by the mononuclear phagocyte system observed in Figure $2 \mathrm{~B}$ and $\mathrm{C}$ most likely as single or multiple liposome-Ab complexes.

In Vitro Evidence for Increased Complement Activation by Doxil in Immunized Pig Sera. The rise of sC5b-9 during massive cardiopulmonary distress in immunized animals (Figure 4A) was significant, but relatively small (2-fold), so we sought other, independent evidence for complement activation by Doxil in pig blood. As shown in Figure 6, incubation of Doxil

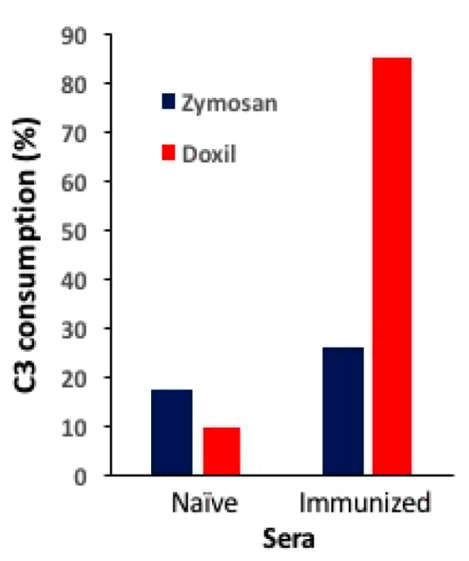

Figure 6. Acceleration of complement activation by anti-PEG IgM in pig serum in vitro. Doxil $(3 \mathrm{mg} \mathrm{PL} / \mathrm{mL})$ and zymosan $(0.3 \mathrm{mg} / \mathrm{mL})$ were incubated with the serum of a Doxebo-immunized pig (titer: 36,843 ) and that of a control (naïve) pig (titer: 122 ) at $37^{\circ} \mathrm{C}$ for 45 min, and $\mathrm{C} 3$ consumption was measured by Quidel's Pan $\mathrm{C} 3$ assay. Immunization was the same as in Figure 1.

with immunized $v s$ naïve pig serum led to significantly increased C3 consumption in the immune serum. Although the effect of zymosan was also higher in the latter serum, the relative change was much greater with Doxil, which is consistent with classical pathway complement activation due to anti-PEG IgM binding.

\section{CONCLUSIONS}

Mechanism of HSR to PEGylated Nanomedicines: Piecing the Steps Together. On the basis of a long list of evidence for a causal role of complement activation in certain liposome-induced HSRs (Supplement Table 1) we proposed earlier that CARPA represents a major mechanism of infusion reactions to a variety of nanoparticulate drugs and agents. ${ }^{21,22}$ However, recently, the role of complement in infusion reactions has been questioned ${ }^{23}$ on the basis of an experiment that showed no complement activation by a polystyrene nanoparticle in pig blood in vitro, although it caused pulmonary reactions in pigs in vivo. ${ }^{24}$ Although the validity of these in vitro data and the generalization of conclusions was debated, ${ }^{25,26}$ the ultimate question, what is the contribution of complement activation to nanoparticle-induced cardiopulmonary distress of pigs vis-à-vis other mechanisms, remained open. This question is particularly relevant for Doxil, since it was shown to activate complement in man both in vitro ${ }^{27}$ and in vivo, ${ }^{28}$ but in cancer patients infused with this drug, only strong complement activation could be associated with HSRs. ${ }^{28}$ Furthermore, direct evidence for complement activation in pigs behind the HSRs to Doxil has never been presented previously. Our present data showing significant correlation between pulmonary hypertension and rise of sC5b-9 in the blood of animals sensitized for Ab-mediated C activation represent direct evidence that complement activation can play a causal role in the pigs' cardiopulmonary distress. In 


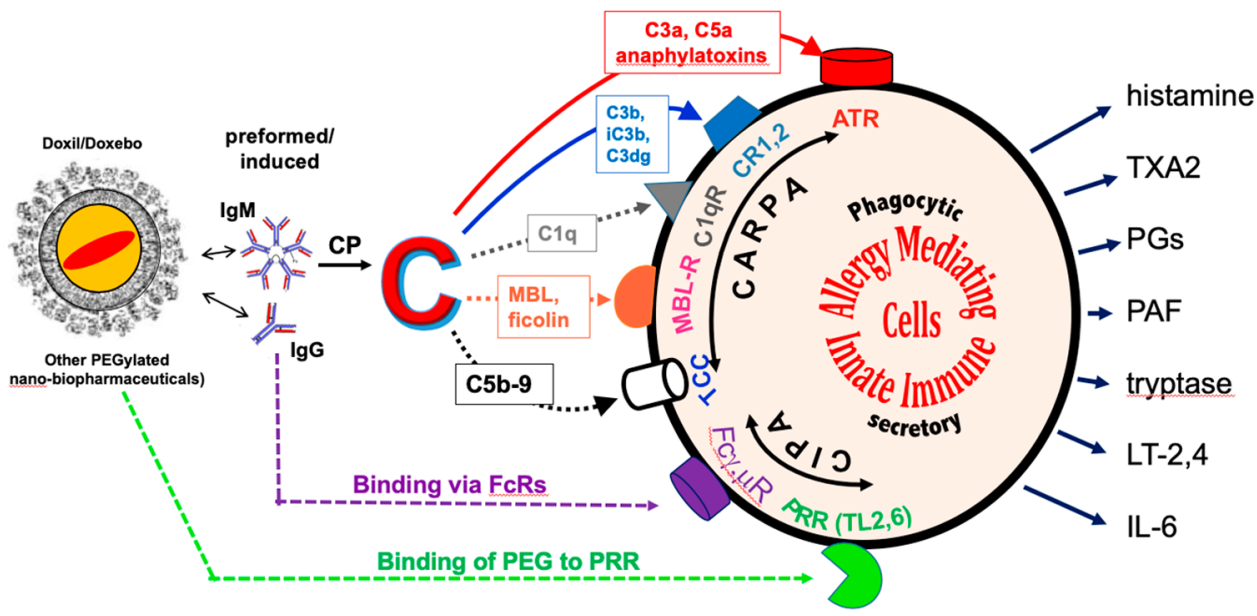

Figure 7. Comprehensive mechanistic scheme of pseudoallergic infusion reactions to Doxil and other PEGylated nanoparticles: known and hypothetical pathways of the reaction cascade. PEGylated liposomes bind anti-PEG Abs, which activate complement via the classical pathway (CP). The liberated complement cleavage products stimulate a variety of innate immune and blood cells (e.g., PIM cells, macrophages, mast cells, basophils, granulocytes, platelets) via different receptors, illustrated with color-coded arrows and surface shapes for different receptors. These signaling pathways represent CARPA, but allergy-mediating phagocytic cells could also be activated without the involvement of complement, referred to as complement-independent pseudoallergy (CIPA). Outward arrows show the known secretory products that can mediate allergic symptoms. Continuous and dotted lines represent known and hypothetical activation mechanisms. Abbreviations: ATR, anaphylatoxin receptor; C3b "opsonin pathway", mediated by C3b, iC3b, and C3d(g) via CR1 (CD35), CR2 (CD21), and CR3 (CD11b/CD18) (blue); "C1q-C1qR pathway" (brown); "MBL/ficolin-MBL-R pathway" (red); "terminal complement complex (C5b-9) direct stimulation pathway" (blue); potential additional stimulation mechanisms include Fc-mediated IgG/IgM binding to Fc $\gamma$ RIIB (CD32)/Fc $\gamma \mathrm{R}$ (CD351) (violet); and PEG binding to patter recognition receptors (PRRs), e.g., Toll-like receptor 2 and/or 6 and/or other PRR, as a consequence of mimicking pathogen-associated molecular patterns (PAMPs) (green).

addition, the acceleration of cardiovascular distress to pseudoanaphylaxis in immunized animals displaying high anti-PEG IgM titer in blood, taken together with the increased C3 conversion by Doxil in immune vs naïve pig serum and the accelerated clearance of anti-PEG IgM on the same time course as complement activation and the cardiopulmonary reaction proceeds, suggests a causal relationship not only between $\mathrm{C}$ activation and pulmonary reaction but also between anti-PEG IgM levels in blood and complement activation. These processes pieced together suggest the comprehensive reaction scheme in Figure 7, illustrating the sequence of reaction steps along an immuno-circulatory axis: first the binding of Abs to liposomes causing complement activation, liberation of anaphylatoxins and other complement cleavage products, and their stimulatory effects on allergy-mediating innate immune cells entailing the secretion of vasoactive and inflammatory mediators, which are responsible for the symptoms of HSRs.

In pigs, pulmonary intravascular macrophages (PIM cells) are likely to play a central role in sensitizing the model, ${ }^{17-20,23-25,39-41}$ but the involvement in HSRs of other anaphylatoxin-sensitive and/or particle-scavenging secretory cells (Kupffer cells, mast cells, dendritic cells, platelets, and granulocytes) cannot be excluded either, just like the contribution of complement-independent stimulation of these cells, referred to as complement-independent pseudoallergy $\left(\right.$ CIPA) ${ }^{25,29}$ (Figure 7). These concurrent stimulatory effects are delineated by the "double-hit" hypothesis proposed for Doxilinduced HSRs in pigs earlier, ${ }^{20}$ which can be extended to "multiple hit" by other potential stimulatory signals on innate immune cells, as illustrated in Figure 7. It should be noted that phagocytosis of nanoparticles, as suggested by the "rapid phagocytic response" hypothesis, ${ }^{23,24}$ represents another possible contributor mechanism of HSRs, but is likely to apply for $\mathrm{C} 3 \mathrm{~b} / \mathrm{iC} 3 \mathrm{~b} / \mathrm{C} 3 \mathrm{dg}$-opsonized nanoparticles ${ }^{26}$ only to the extent that phagocytic cells can internalize them within 2 min. The idea of complement-independent rapid phagocytosis remains to be proven; ${ }^{23}$ however, it is unlikely to apply to PEGylated liposomes, whose phagocytosis is known to be inhibited by the PEG coating.

Explanations for Anti-PEG IgM Clearance. The rapid decline of anti-PEG IgM titer in the plasma of naïve pigs within the first minute after Doxil injection (Figure 2B) may have ex vivo and in vivo reasons, including competitive inhibition of antiPEG IgM capture by free liposomes in the ELISA plate and IgMinduced polyvalent binding of multiple vesicles in vivo, entailing aggregation and capillary sequestration. These possibilities need further investigations. Nevertheless, the subsequent first-order decay of anti-PEG IgM within 10-15 min that can be modeled by a near-perfect logarithmic equation reflects a biological process whose rate is proportional to the amount that is left. In our case this process is most likely scavenging of liposome-IgM complexes by cells of the mononuclear phagocyte system (MPS) with or without phagocytosis, a phenomenon known as accelerated blood clearance $(\mathrm{ABC}) .^{30-35}$

The Concept of "Primary ABC". In ABC, studied for nearly two decades mainly in murine models, ${ }^{30-35}$ the rapid clearance of liposomes has been attributed to the binding of anti-PEG IgM to the vesicles, entailing complement activation, opsonization, and rapid clearance by the MPS. These anti-PEG Abs were shown to be formed in splenic marginal zone B cells via T-cell independent immunization by a prior injection of PEGylated liposomes. ${ }^{32-35}$ It is therefore a fundamental difference between the above "classical" ABC and our observations on rapid antiPEG IgM clearance that the latter process occurs already after the first injection of liposomes; there is no priming (Figure 2B and $\mathrm{C}$ ). Assuming that the anti-PEG IgM undergoing rapid clearance was liposome-bound, the finding suggests that preexisting, naturally occurring anti-PEG IgM can similarly 
remove liposomes as the induced Abs in "classical" ABC. Thus, the rapid decay of anti-PEG IgM shown in Figure 2B and C may reflect "primary" $A B C$ with many of the attributes of "secondary" $\mathrm{ABC}$. Accordingly, primary $\mathrm{ABC}$ and CARPA may represent "two sides of the same coin", ${ }^{35}$ raising the question of why HSR was never reported during secondary $\mathrm{ABC}$ in rats and mice. The most likely explanation is that these species have orders of magnitude lower sensitivity for HSRs than pigs or hypersensitive human. ${ }^{29,36}$ It is the high sensitivity of the pig model for HSRs that enabled uncovering of this duality.

Direct Evidence for IgM Binding to Doxil. The specific binding between PEGylated liposomes and anti-PEG IgM during secondary $\mathrm{ABC}$ in murine models was inferred, among others, from flow cytometric analysis of the binding of fluorescence-labeled PEGylated liposomes to liposome-primed spleen cells in vitro, which correlated with the production of antiPEG antibodies, determined by ELISA. ${ }^{37,38}$ In an effort to reproduce this observation for the case of pig splenocytes obtained from naive and immunized pigs in our study, we carried out the flow cytometry experiments shown in Figure 5. The data indicated the presence of liposome-bound anti-PEG IgM on splenic B cells (even in naïve animals), whose number significantly increased after immunization, leading to increased binding of Doxil. These ex vivo observations in cell culture are in keeping with all in vivo results in pigs and point to splenic B cells as a likely source of anti-PEG IgM in pigs as well.

Criticism of the Porcine Model. The pig model used in this study has recently been claimed to be irrelevant to the average human patient and is therefore misleading for assessing the risk of nanomedicine-induced HSRs. ${ }^{23,39-41}$ The reasons for this view included the difference of HSR rate in pigs and the average human, different underlying immune mechanisms, and the "globality" of cardiopulmonary reactions. To clarify possible misunderstandings that led to the above conclusions, it seems important to re-emphasize ${ }^{18,19,25}$ that the pig model is not a traditional immune toxicology model where the toxicities of drugs on the immune system are assessed in healthy animals to predict possible adverse immune effects in immunologically normal humans. Like other animal models of allergy, the pig model is an allergic disease model, i.e., that of severe hypersensitivity of a small percentage of people to certain i.v. administered nanopharmaceuticals. Regardless of the underlying mechanism, the concordance with human symptoms (see below) enables the model to serve for identifying the hazard of hypersensitivity reaction to the tested drug in the case of hypersensitive patients. Also, certain cardiopulmonary and other physiological changes are quantitative and highly reproducible within the dynamic window of the test, where the adverse response is dose dependent. This allows using the model for the assessment of the risk that the tested drugs might cause HSR, to study the mechanism of these reactions and develop methods for their prevention.

Clinical Relevance of the Porcine CARPA Model. In keeping with the above claims on human relevance, our observations in the pig model show significant concordance with some recent clinical trial data. Namely, the "RADAR" and "REGULATE-PCI" trials with pegnivacogin (Revolixys kit) ${ }^{42,43}$ were stopped because of life-threatening HSRs. The tested PEGylated aptamer caused anaphylaxis in 3/640 (0.46\%) and $10 / 1605(0.62 \%)$ patients, and reactions were observed exclusively in those who had high levels of preformed antiPEG antibodies. ${ }^{42,43}$ Importantly, the indications of complement activation (rises of $\mathrm{C} 3 \mathrm{a}, \mathrm{Bb}$, drop of $\mathrm{CH} 50$ ) included $\mathrm{C} 4 \mathrm{~d}$, a biomarker of classical pathway complement activation. ${ }^{42,43}$ In agreement with the conclusion in the present study, the authors of the above-mentioned clinical study suggested anti-PEG Abtriggered complement activation (i.e., CARPA) as the underlying cause of anaphylactic reactions, although they assign the reactions to anti-PEG IgG, rather than anti-PEG IgM (which was not measured). In another clinical study on the frequent and severe HSRs to pegloticase (Krystexxa), a PEGylated recombinant porcine enzyme (uricase) used for the treatment of refractory gout, the HSRs were shown to be correlated with the preexisting and induced anti-PEG Abs and rapid loss of the drug's efficacy. ${ }^{14,44}$ Yet another testimony to the clinical relevance of the pig model is the recent FDA approval of a transthyretin-directed small inhibitory RNA-containing nanoparticle (patisiran, Onpattro), whose safe administration protocol was developed, in part, using the porcine CARPA model. $^{45}$

Perspective. This study provided experimental support for the concept that PEGylated liposome-induced CARPA, whose worst manifestation is pseudo-anaphylactic shock, proceeds along the complement-circulatory system axis, via acute cascadic activation of innate humoral and cellular immunity. This information may help to better understand and, hence, more efficiently predict and prevent HSRs to PEGylated and other nanopharmaceuticals. Furthermore, the listed evidence of concordance between the physiological and immune responses to PEGylated nanoparticles in pigs and humans, as well as the example of using the model by the biopharma industry, provides strong support for the human relevance and use of the porcine CARPA model for hazard identification in preclinical safety studies.

\section{MATERIALS AND METHODS}

Drugs and Chemicals. Commercial Doxil (Caelyx) was obtained from the pharmacy of Semmelweis University (in this paper we refer to Doxil, as the two names represent the same drug). It contains doxorubicin, $2 \mathrm{mg} / \mathrm{mL}(4.22 \mathrm{mM})$; fully hydrogenated soy phosphatidylcholine (HSPC), $9.58 \mathrm{mg} / \mathrm{mL}$; cholesterol, $3.19 \mathrm{mg}$ / mL; 2K-PEG-DSPE ( $N$-carbamylpoly(ethylene glycol methyl ether)1,2-distearoyl-sn-glycerol-3-phosphoethanolamine triethylammonium salt with a polyethylene glycol moiety of average molecular mass of $2000 \mathrm{Da}, 3.19 \mathrm{mg} / \mathrm{mL}$; ammonium sulfate, $\sim 0.2 \mathrm{mg} / \mathrm{mL}$; histidine $(10$ $\mathrm{mM}, \mathrm{pH} 6.5$ ); and sucrose (10\%). Total phospholipid: $12.8 \mathrm{mg} / \mathrm{mL}$ (13.3 mM). Cholesterol was purchased from Avanti Polar Lipids Inc. (Alabaster, AL, USA), mPEG2000-DSPE was from NOF, and HSPC was from Lipoid Inc. (Ludwigshafen, Germany). All lipids had a purity of $\geq 97 \%$. Zymosan, Dulbecco's phosphate-buffered saline (PBS) without $\mathrm{Ca}^{2+} / \mathrm{Mg}^{2+}$ and bovine calf serum, and biotin-labeled goat polyclonal anti-IgM were from Sigma Chemical Co. (St. Louis, MO, USA). Doxorubicin was from TEVA Pharmaceuticals (Petach Tikva, Israel). Streptavidine-FITC was from Molecular Probes. FACS Lysing solution and paraformaldehyde were from BD. The MicroVue Pan C3 kit (Quidel Corp, cat. no. 20261) was obtained from TECOMedical (Sissach, Switzerland). Anti-PEG mouse monoclonal IgM (ANPEG-1) was obtained from ANP Technologies, Inc. (Newark, DE, USA).

Preparation of Doxebo. The preparation and characteristics of Doxebo were described earlier. ${ }^{20}$ In brief, the freeze-dried lipid components of Doxil (listed above) were hydrated in $10 \mathrm{~mL}$ sterile pyrogen-free normal saline by vortexing for $2-3 \mathrm{~min}$ at $70{ }^{\circ} \mathrm{C}$ to form multilamellar vesicles (MLVs). The MLVs were downsized through 0.4 and $0.1 \mu \mathrm{m}$ polycarbonate filters in two steps, 10 times through each, using a $10 \mathrm{~mL}$ extruder barrel from Northern Lipids (Vancouver, British Columbia, Canada) at $62{ }^{\circ} \mathrm{C}$. Liposomes were suspended in $0.15 \mathrm{M}$ $\mathrm{NaCl} / 10 \mathrm{mM}$ histidine buffer ( $\mathrm{pH}$ 6.5). The size distribution ( $Z$ average: $81.17 \mathrm{~nm}$ ) and phospholipid concentration of Doxebo (12.6 $\mathrm{mg} / \mathrm{mL}$ ) were determined as described. ${ }^{20}$ 
Determination of Bacterial Endotoxin (LPS) in Liposomes. The LPS content of liposomes prepared for this study was determined by a limulus amebocyte lysate assay (PYROGENT Plus, Cambrex Bio Science, East Rutherford, NJ, USA), after dissolving ( $96 \%$ ethanol) and separating (ultrafiltration using a $20 \mathrm{kDa}$ cutoff membrane) the lipids from LPS. Acceptance criteria as pyrogen-free were $\leq 0.5 \mathrm{EU} / \mathrm{mL}$ (0.01-0.25 ng LPS $/ \mathrm{mL}$ ).

Pig Studies. The details and evaluation of the porcine "CARPA model" were described in several studies earlier. ${ }^{17-20,44}$ In brief, mixed breed male Yorkshire/Hungarian White Landrace pigs (2-3 months old, $18-22 \mathrm{~kg}$ ) were obtained from the Animal Breeding and Nutrition Research Institute, Herceghalom, Hungary. Animals were sedated with Calypsol/Xilazine and then anesthetized with isoflurane $\left(2-3 \%\right.$ in $\left.\mathrm{O}_{2}\right)$. Intubation was performed with endotracheal tubes to maintain free airways and to enable controlled ventilation if necessary. The animals were breathing spontaneously during the experiments. Surgery was done after povidone iodine (10\%) disinfection of the skin. In order to measure the PAP, a Swan-Ganz catheter (AI-07124, 5 Fr. $110 \mathrm{~cm}$, Arrow Internat Inc.) was introduced into the pulmonary artery via the right external jugular vein. Additional catheters were placed into the left femoral artery to record the systemic arterial pressure (SAP), into the left external jugular vein for saline and drug administration, and into the left femoral vein for blood sampling.

The test samples (liposome suspensions) and zymosan were injected into the animals as a bolus $(<10 \mathrm{~s})$ into the pulmonary artery or in an infusion via the left external jugular vein. The experiment typically involved bolus injection of $0.1 \mathrm{mg} \mathrm{PL} / \mathrm{kg}$ Doxebo, followed by a similar dose of Doxil $(0.1 \mathrm{mg}$ PL/kg), then a larger dose of Doxil, and, finally, $0.1 \mathrm{mg} / \mathrm{kg}$ zymosan as positive control. At the end of the experiments animals were sacrificed with Euthasol and concentrated potassium chloride.

Monitoring of Different Physiological Changes during CARPA. The area under the PAP and SAP change (AUC) was calculated by summing the PAP and SAP $\mathrm{mm} \mathrm{Hg}$ values according to the formula

$$
\sum_{n=1}^{x} \operatorname{abs}\left(\frac{\mathrm{PAP}_{n}+\mathrm{PAP}_{n+1}}{2}-\mathrm{PAP}_{0}\right)
$$

where " $n$ " represent time and " $x$ " is the last minute (typically 10) of observation. $\mathrm{PAP}_{0}$ is the PAP value immediately before treatment (equivalent with baseline).

Pretreatment (Immunization, Sensitization) of Pigs. Immunization with Doxebo on day 0 consisted of infusion of Doxebo $(0.1 \mathrm{mg}$ $\mathrm{PL} / \mathrm{kg}$ ) in pigs via their ear vein (suspended in $20 \mathrm{~mL}$ of saline) at a speed of $1 \mathrm{~mL} / \mathrm{min}$ over $20 \mathrm{~min}$.

Blood Samples. Blood samples for $\mathrm{Ab}$ analysis were taken from the ear vein before pretreatment and then at different times specified in the Results. Anticoagulation was done with either EDTA or hirudin, by using the corresponding hirudin- or $\mathrm{K}_{3}$-EDTA tubes.

ELISA of Porcine sC5b-9. Porcine sC5b-9 determination was done as described earlier. ${ }^{46}$ In brief, microtiter plates were coated with mouse anti-human sC5b-9 ascites (clone aE11) and incubated for $1 \mathrm{~h}$ with plasma containing $10 \mathrm{mM}$ EDTA. The second Ab, biotinylated mouse anti-human C6 (Quidel A219), was stained with streptavidinhorseradish peroxidase (HRP) using ABTS and $\mathrm{H}_{2} \mathrm{O}_{2}$ substrate.

ELISA of Anti-PEG Antibodies. Polysorp (Nunc) plates were coated with $1.25 \mu \mathrm{g} /$ well DSPE-PEG2000 in $100 \mu \mathrm{L}$ of bicarbonate buffer $(4.46 \mu \mathrm{M})(\mathrm{pH} \sim 9.0)$ overnight at $4{ }^{\circ} \mathrm{C}$, followed by blocking of the wells with $150 \mu \mathrm{L}$ of PBS $/ 0.05 \%$ Tween- $20+2 \%$ bovine serum albumin (BSA) at $37^{\circ} \mathrm{C}$ for $1.5 \mathrm{~h}$. Before and after blocking, wells were washed two and three times with $300 \mu \mathrm{L}$ of wash buffer containing PBS/0.05\% Tween-20 for $1 \mathrm{~min}$, respectively. The EDTA-anticoagulated plasma samples were diluted by PBS $/ 0.05 \%$ Tween- $20+$ $1 \%$ BSA in the 10-19 500-fold range and incubated in the wells for 1.5 $\mathrm{h}$ at $37^{\circ} \mathrm{C}$, with slow shaking. Wells were washed five times with $300 \mu \mathrm{L}$ of wash buffer for $1 \mathrm{~min}$. After staining with $100 \mu \mathrm{L}$ of HRP-conjugated anti-porcine IgM (2000× dilution, Sigma) or IgG $(800 \times$ dilution, Sigma) for $1 \mathrm{~h}$, wells were washed again five times with wash buffer as mentioned. The antibodies were stained by incubation with $100 \mu \mathrm{L}$ of substrate solution (Neogen) containing 3,3',5,5'-tetramethylbenzidine (TMB) and hydrogen peroxide for $15 \mathrm{~min}$ in dark. The reaction was stopped with $50 \mu \mathrm{L}$ of $2 \mathrm{~N} \mathrm{H}_{2} \mathrm{SO}_{4}$, and $A_{450}$ was read with a Fluostar Omega 96-well plate reader (BMG Labtech, Ortenberg, Germany). The titer unit was defined as the dilution at which the blank-corrected OD was 0.1. The ELISA was validated as described in the Supporting Information (Figure S1).

Binding of Doxil to Splenic IgM Positive B Cells. A Doxeboimmunized pig (see above) and a naïve pig were sacrificed 9 days after immunization, and their spleens were removed for flow cytometric assessment of the binding of Doxil to anti-PEG IgM+ B cells. Single-cell suspensions from spleen tissue slices were prepared by gentle homogenization in Dulbecco's PBS without $\mathrm{Ca}^{2+} / \mathrm{Mg}^{2+}$ supplemented with $2 \%$ bovine calf serum and $2 \mathrm{mM}$ EDTA. The latter medium was sterilized by filtration, and the cell suspension was homogenized by aspirations via a $21 \mathrm{G}$ needle. Cells were centrifuged $\left(20^{\circ} \mathrm{C}, 500 \mathrm{~g}, 8\right.$ $\mathrm{min}$ ) and washed and suspended in the medium at $10^{6}$ cells $/ \mathrm{mL}$. This was followed by incubation with biotin-labeled anti-IgM and Doxil for $30 \mathrm{~min}$ at $4{ }^{\circ} \mathrm{C}$. After washing, IgM-labeled cells were stained by streptavidin-FITC for $20 \mathrm{~min}$ at room temperature (RT). Following repeated washing, red blood cells were lysed by FACS Lysing solution for $10 \mathrm{~min}$ at RT. Finally, after a final washing, cells were resuspended in $1 \%$ paraformaldehyde. FACS analysis was done in a FACScan (BD), and the data were analyzed by Kaluza software (Beckman Coulter). In control staining tubes, cells were incubated without Doxil or without Doxil and anti-IgM (just with biotin-FITC).

Measurement of Pig C 3 Consumption in Vitro by ELISA. Pig sera obtained from a naïve and an immunized animal (day 7) were incubated with Doxil and zymosan (activator/serum volume ratio was $1 / 3$ ) for $45 \mathrm{~min}$ at $37{ }^{\circ} \mathrm{C}$ with shaking. Incubation was stopped by adding 10 vol ELISA sample diluent complemented with $10 \mathrm{mM}$ EDTA, and the samples were measured for porcine C3 level using the MicroVue Pan-Specific C3 reagent kit (Quidel Corp) according to the manufacturer's instructions. The 45 min values were compared to the baseline, i.e., similar samples analyzed immediately after mixing the activators with the serum $(0 \mathrm{~min})$. Consumption was calculated by 100 - $\left(\mathrm{OD}_{45 \text { min }} / \mathrm{OD}_{0 \text { min }} \times 100\right)$, where $\mathrm{OD}_{45 \text { min }}$ and $\mathrm{OD}_{0 \text { min }}$ are absorbances at $450 \mathrm{~nm}$ at the specified times.

Statistical Methods. Normality was tested by the KolmogorovSmirnov test. The anti-PEG Ab titers and PAP values at all time points were compared to their baseline $(0 \mathrm{~min})$, and the significance of differences was determined by nonparametric Kruskal-Wallis and Friedman tests, followed by Dunn's multiple comparisons, respectively. To study the correlation between changes in the sC5b-9 values and PAP AUC values in response to Doxebo/Doxil treatments 4-6 weeks following immunization, we used Pearson analysis, preceded by testing for normal distribution and outliers by Kolmogorov-Smirnov and Grubb's tests, respectively. A $P$-value of less than 0.05 was considered to be statistically significant. Statistical analysis was performed by GraphPad Prism software (GraphPad Software, La Jolla, CA, USA).

\section{ASSOCIATED CONTENT}

\section{Supporting Information}

The Supporting Information is available free of charge on the ACS Publications website at DOI: 10.1021/acsnano.9b03942.

Experiments validating the anti-PEG antibody ELISA; a table listing the evidence that complement activation plays a role in liposome-induced hypersensitivity reactions $(\mathrm{PDF})$

\section{AUTHOR INFORMATION}

\section{Corresponding Author}

*E-mail: jszebeni@seroscience.com.

ORCID $\odot$

János Szebeni: 0000-0003-1738-797X 


\section{Author Contributions}

G.K., R.U., Y.B., and J.S. designed the experiments, analyzed the data, and wrote the paper. G.K., T.M., D.I.V., L.D., E.O., R.U., and Y.B. conducted and R.L. helped in organizing the experiments. All authors discussed the progress of research and reviewed the manuscript.

\section{Author Contributions}

$\square$ Y.B. and J.S. are equal senior authors.

\section{Notes}

The authors declare no competing financial interest.

\section{ACKNOWLEDGMENTS}

The authors acknowledge the following financial support: the Barenholz Fund (Israel); the European Union Seventh Framework Program grant no. 309820 (NanoAthero) and no. 602923 (Theraglio); the Hungarian Scientific Research Fund (OTKA/ NFKI) project K-113164, the Applied Materials and Nanotechnology Center of Excellence, Miskolc University; the Norwegian Council on Cardiovascular Disease, the Odd Fellow Foundation, the Simon Fougner Hartmann Family Fund, and the European Community's Seventh Framework Programme under grant agreement no. 602699 (DIREKT). The authors thank Maria Velkei for laboratory assistance.

\section{REFERENCES}

(1) Cheifetz, A.; Smedley, M.; Martin, S.; Reiter, M.; Leone, G.; Mayer, L.; Plevy, S. The Incidence and Management of Infusion Reactions to Infliximab: a Large Center Experience. Am. J. Gastroenterol. 2003, 98, 1315-1324.

(2) Mayer, L.; Young, Y. Infusion Reactions and Their Management. Gastroenterol Clin North Am. 2006, 35, 857-866.

(3) Lenz, H. J. Management and Preparedness for Infusion and Hypersensitivity Reactions. Oncologist 2007, 12, 601-609.

(4) Vogel, W. H. Infusion Reactions: Diagnosis, Assessment, and Management. Clin J. Oncol Nurs 2010, 14, E10-E21.

(5) Ring, J.; Beyer, K.; Biedermann, T.; Bircher, A.; Duda, D.; Fischer, J.; Friedrichs, F.; Fuchs, T.; Gieler, U.; Jakob, T.; Klimek, L.; Lange, L.; Merk, H. F.; Niggemann, B.; Pfaar, O.; Przybilla, B.; Ruëff, F.; Rietschel, E.; Schnadt, S.; Seifert, R.; et al. Guideline for Acute Therapy and Management of Anaphylaxis. Allergo J. Int. 2014, 23, 96-112.

(6) Ingen-Housz-Oro, S.; Pham-Ledard, A.; Brice, P.; Lebrun-Vignes, B.; Zehou, O.; Reitter, D.; Ram-Wolff, C.; Dupin, N.; Bagot, M.; Chosidow, O.; Beylot-Barry, M. Immediate Hypersensitivity Reaction to PEGylated Liposomal Doxorubicin: Management and Outcome in Four Patients. Eur. J. Dermatol 2017, 27, 271-274.

(7) Szebeni, J.; Simberg, D.; Gonzalez-Fernandez, A.; Barenholz, Y.; Dobrovolskaia, M. A. Roadmap and Strategy for Overcoming Infusion Reactions to Nanomedicines. Nat. Nanotechnol. 2018, 13, 1100-1108.

(8) Doxil Prescribing Information. https://www.accessdata.fda.gov/ drugsatfda_docs/label/2007/050718s029lbl.pdf. Accessed July 17, 2019.

(9) Dadla, A.; Tannenbaum, S.; Yates, B.; Holle, L. Delayed Hypersensitivity Reaction Related to the Use of Pegfilgrastim. J. Oncol. Pharm. Pract. 2015, 21, 474-477.

(10) Davila Fajardo, C. L.; Pena Ortega, M.; Cabeza Barrera, J.; Prados Garrido, M. D. Methoxy Polyethylene Glycol-Epoetin Beta (Mircera) in the Treatment of a Patient with Chronic Kidney Disease Presenting Late-Onset Hypersensitivity to Other Epoetins. Nefrologia 2010, 30, 372-373.

(11) Jivi, A. Antihemophilic Factor (Recombinant), Prescribing Information. https://www.fda.gov/media/94470/download (accessed July 17,2019$)$.

(12) Palynziq (pegvaliase-pqpz), Prescribing Information.https:// www.palynziq.com/prescribinginformation.pdf (accessed July 17, 2019).
(13) Hermanson, T.; Bennett, C. L.; Macdougall, I. C. Peginesatide for the Treatment of Anemia Due To Chronic Kidney Disease - An Unfulfilled Promise. Expert Opin. Drug Saf. 2016, 15, 1421-1426.

(14) Calabrese, L. H.; Kavanaugh, A.; Yeo, A. E.; Lipsky, P. E. Frequency, Distribution and Immunologic Nature of Infusion Reactions in Subjects Receiving Pegloticase For Chronic Refractory Gout. Arthritis Res. Ther. 2017, 19, 191-197.

(15) Povsic, T. J.; Vavalle, J. P.; Aberle, L. H.; Kasprzak, J. D.; Cohen, M. G.; Mehran, R.; Bode, C.; Buller, C. E.; Montalescot, G.; Cornel, J. H.; Rynkiewicz, A.; Ring, M. E.; Zeymer, U.; Natarajan, M.; Delarche, N.; Zelenkofske, S. L.; Becker, R. C.; Alexander, J. H. A Phase 2, Randomized, Partially Blinded, Active-Controlled Study Assessing The Efficacy and Safety of Variable Anticoagulation Reversal Using the REG1 System in Patients with Acute Coronary Syndromes: Results of the RADAR Trial. Eur. Heart J. 2013, 34, 2481-2489.

(16) Szebeni, J.; Baranyi, L.; Savay, S.; Bodo, M.; Milosevits, J.; Alving, C. R.; Bunger, R. Complement Activation-Related Cardiac Anaphylaxis in Pigs: Role of C5a Anaphylatoxin and Adenosine in LiposomeInduced Abnormalities in ECG and Heart Function. Am. J. Physiol Heart Circ Physiol 2006, 290, 1050-1058.

(17) Szebeni, J.; Bedocs, P.; Csukas, D.; Rosivall, L.; Bunger, R.; Urbanics, R. A. Porcine Model of Complement-Mediated Infusion Reactions to Drug Carrier Nanosystems and Other Medicines. Adv. Drug Delivery Rev. 2012, 64, 1706-1716.

(18) Urbanics, R.; Szebeni, J. Lessons Learned from the Porcine CARPA Model: Constant and Variable Responses to Different Nanomedicines and Administration Protocols. Eur. J. Nanomed. 2015, 7, 219-231.

(19) Szebeni, J.; Bedocs, P.; Dezsi, L.; Urbanics, R. A. Porcine Model of Complement Activation-Related Pseudoallergy to Nano-Pharmaceuticals: Pros and Cons of Translation to a Preclinical Safety Test. Prec Nanomed 2018, 1, 63-73.

(20) Szebeni, J.; Bedocs, P.; Urbanics, R.; Bunger, R.; Rosivall, L.; Toth, M.; Barenholz, Y. Prevention of Infusion Reactions to PEGylated Liposomal Doxorubicin via Tachyphylaxis Induction by Placebo Vesicles: a Porcine Model. J. Controlled Release 2012, 160, 382-387.

(21) Szebeni, J. Complement Activation-Related Pseudoallergy: a New Class of Drug-Induced Acute Immune Toxicity. Toxicology 2005, 216, 106-121.

(22) Szebeni, J. Complement Activation-Related Pseudoallergy: a Stress Reaction in Blood Triggered by Nanomedicines and Biologicals. Mol. Immunol. 2014, 61, 163-173.

(23) Moghimi, S. M. Nanomedicine Safety in Preclinical and Clinical Development: Focus on Idiosyncratic Injection/Infusion Reactions. Drug Discovery Today 2018, 23, 1034-1042.

(24) Wibroe, P. P.; Anselmo, A. C.; Nilsson, P. H.; Sarode, A.; Gupta, V.; Urbanics, R.; Szebeni, J.; Hunter, A. C.; Mitragotri, S.; Mollnes, T. E.; Moghimi, S. M. Bypassing Adverse Injection Reactions to Nanoparticles Through Shape Modification and Attachment to Erythrocytes. Nat. Nanotechnol. 2017, 12, 589-594.

(25) Szebeni, J. Mechanism of Nanoparticle-Induced Hypersensitivity in Pigs: Complement or Not Complement? Drug Discovery Today 2018, 23, 487-492.

(26) Meszaros, T.; Kozma, G. T.; Shimizu, T.; Miyahara, K.; Turjeman, K.; Ishida, T.; Barenholz, Y.; Urbanics, R.; Szebeni, J. Involvement of Complement Activation in the Pulmonary Vasoactivity of Polystyrene Nanoparticles in Pigs: Unique Surface Properties Underlying Alternative Pathway Activation and Instant Opsonization. Int. J. Nanomed. 2018, 13, 6345-6357.

(27) Szebeni, J.; Baranyi, L.; Savay, S.; Lutz, H. U.; Jelezarova, E.; Bunger, R.; Alving, C. R. The Role of Complement Activation in Hypersensitivity to PEGylated Liposomal Doxorubicin (Doxil®). J. Liposome Res. 2000, 10, 467-481.

(28) Chanan-Khan, A.; Szebeni, J.; Savay, S.; Liebes, L.; Rafique, N. M.; Alving, C. R.; Muggia, F. M. Complement Activation Following First Exposure to PEGylated Liposomal Doxorubicin (Doxil): Possible Role in Hypersensitivity Reactions. Ann. Oncol 2003, 14, 1430-1437.

(29) Orfi, E.; Meszaros, T.; Hennies, M.; Fulop, T.; Dezsi, L.; Nardocci, A.; Rosivall, L.; Hamar, P.; Neun, B. W.; Dobrovolskaia, M. 
A.; Szebeni, J.; Szenasi, G. Acute Physiological Changes Caused by Complement Activators and Amphotericin B-Containing Liposomes in Mice. Int. J. Nanomed. 2019, 14, 1563-1573.

(30) Dams, E. T.; Laverman, P.; Oyen, W. J.; Storm, G.; Scherphof, G. L.; van Der Meer, J. W.; Corstens, F. H.; Boerman, O. C. Accelerated Blood Clearance and Altered Biodistribution of Repeated Injections of Sterically Stabilized Liposomes. J. Pharmacol Exp Ther 2000, 292, 1071-1079.

(31) Ishida, T.; Harada, M.; Wang, X. Y.; Ichihara, M.; Irimura, K.; Kiwada, H. Accelerated Blood Clearance of PEGylated Liposomes Following Preceding Liposome Injection: Effects of Lipid Dose and PEG Surface-Density and Chain Length of the First-Dose Liposomes. J. Controlled Release 2005, 105, 305-317.

(32) Koide, H.; Asai, T.; Hatanaka, K.; Akai, S.; Ishii, T.; Kenjo, E.; Ishida, T.; Kiwada, H.; Tsukada, H.; Oku, N. T Cell-Independent B Cell Response Is Responsible for $\mathrm{ABC}$ Phenomenon Induced by Repeated Injection of PEGylated Liposomes. Int. J. Pharm. 2010, 392, 218-223.

(33) Shimizu, T.; Mima, Y.; Hashimoto, Y.; Ukawa, M.; Ando, H.; Kiwada, H.; Ishida, T. Anti-PEG IgM and Complement System Are Required for the Association of Second Doses of PEGylated Liposomes with Splenic Marginal Zone B Cells. Immunobiology 2015, 220, 11511160.

(34) Hashimoto, Y.; Shimizu, T.; Abu Lila, A. S.; Ishida, T.; Kiwada, $\mathrm{H}$. Relationship between the Concentration of Anti-Polyethylene Glycol (PEG) Immunoglobulin M (IgM) and the Intensity of the Accelerated Blood Clearance (ABC) Phenomenon against PEGylated Liposomes in Mice. Biol. Pharm. Bull. 2015, 38, 417-424.

(35) Abu Lila, A. S.; Szebeni, J.; Ishida, T. Accelerated Blood Clearance Phenomenon and Complement Activation-Related Pseudoallergy: Two Sides of the Same Coin. In Immune Effects of Biopharmaceuticals and Nanomedicines; Bawa, R.; Szebeni, J.; Webster, T. J.; Audette, G. F., Eds.; Pan Stanford Publishing Pte. Ltd.: Singapore, 2018; pp 771-800.

(36) Dezsi, L.; Fulop, T.; Meszaros, T.; Szenasi, G.; Urbanics, R.; Vazsonyi, C.; Orfi, E.; Rosivall, L.; Nemes, R.; Kok, R. J.; Metselaar, J. M.; Storm, G.; Szebeni, J. Features of Complement Activation-Related Pseudoallergy to Liposomes with Different Surface Charge and PEGylation: Comparison of the Porcine and Rat Responses. J. Controlled Release 2014, 195, 2-10.

(37) Abu Lila, A. S.; Ichihara, M.; Shimizu, T.; Ishida, T.; Kiwada, H. Ex Vivo/In Vitro Anti-Polyethylene Glycol (PEG) Immunoglobulin M Production from Murine Splenic B Cells Stimulated by PEGylated Liposome. Biol. Pharm. Bull. 2013, 36, 1842-1348.

(38) Shimizu, T.; Abu Lila, A. S.; Awata, M.; Kubo, Y.; Mima, Y.; Hashimoto, Y.; Ando, H.; Okuhira, K.; Ishima, Y.; Ishida, T. A Cell Assay for Detecting Anti-PEG Immune Response against PEGModified Therapeutics. Pharm. Res. 2018, 35, 223-233.

(39) Skotland, T. Injection of Nanoparticles into Cloven-Hoof Animals: Asking for Trouble? Theranostics 2017, 7, 4877-4878.

(40) Moghimi, S. M.; Simberg, D. Translational Gaps in Animal Models of Human Infusion Reactions to Nanomedicines. Nanomedicine (London, U. K.) 2018, 13, 973-975.

(41) Moghimi, S. M.; Simberg, D.; Skotland, T.; Yaghmur, A.; Hunter, C. The Interplay Between Blood Proteins, Complement, and Macrophages on Nanomedicine Performance and Responses. J. Pharmacol. Exp. Ther. 2019, 370.

(42) Ganson, N. J.; Povsic, T. J.; Sullenger, B. A.; Alexander, J. H.; Zelenkofske, S. L.; Sailstad, J. M.; Rusconi, C. P.; Hershfield, M. S. PreExisting Anti-Polyethylene Glycol Antibody Linked to First-Exposure Allergic Reactions to Pegnivacogin, a PEGylated RNA Aptamer. J. Allergy Clin. Immunol. 2016, 137, 1610-1613.

(43) Povsic, T. J.; Lawrence, M. G.; Lincoff, A. M.; Mehran, R.; Rusconi, C. P.; Zelenkofske, S. L.; Huang, Z.; Sailstad, J.; Armstrong, P. W.; Steg, P. G.; Bode, C.; Becker, R. C.; Alexander, J. H.; Adkinson, N. F.; Levinson, A. I. Pre-Existing Anti-Polyethylene Glycol Antibodies Are Associated with Severe Immediate Allergic Reactions to Pegnivacogin, a PEGylated Aptamer. J. Allergy Clin. Immunol. 2016, $138,1712-1715$.
(44) Hershfield, M. S.; Ganson, N. J.; Kelly, S. J.; Scarlett, E. L.; Jaggers, D. A.; Sundy, J. S. Induced and Pre-Existing Anti-Polyethylene Glycol Antibody in a Trial of Every 3-Week Dosing of Pegloticase for Refractory Gout, Including in Organ Transplant Recipients. Arthritis Res. Ther 2014, 16, R63-73.

(45) Kasperovic, P. Dosages and Methods for Delivering Lipid Formulated Nucleic Acid Molecules. U.S. patent application 20160122759, 2016.

(46) Jansen, J. H.; Hogasen, K.; Mollnes, T. E. Extensive Complement Activation in Hereditary Porcine Membranoproliferative Glomerulonephritis Type II (Porcine Dense Deposit Disease). Am. J. Pathol. 1993, $143,1356-1365$. 